\title{
Genomic patterns and characterizations of chromosomally-encoded mcr-1 in Escherichia coli populations
}

\author{
Cong Shen 1,2, Lan-Lan Zhong 1,2, Furong Ma33, Mohamed Abd El-Gawad El-Sayed Ahmed ${ }^{1,2,4}$, Yohei Doi ${ }^{5,6}$, \\ Guili Zhang ${ }^{1,2}$, Yang Liu ${ }^{1,2}$, Songyin Huang ${ }^{7}$, Hong-Yu Li ${ }^{7}$, Liyan Zhang ${ }^{8}$, Kang Liao ${ }^{9}$, Yong Xia ${ }^{3}$, Min Dai ${ }^{10}$, \\ Bin Yan $^{11}$ and Guo-Bao Tian ${ }^{1,2,12^{*}}$ (1)
}

\begin{abstract}
The emergence and transmission of the mobile colistin resistance gene ( $m c r-1)$ threatened the extensive use of polymyxin antimicrobials. Accumulated evidence showed that the banning of colistin additive in livestock feed efficiently reduce $\mathrm{mcr}-1$ prevalence, not only in animals but also in humans and environments. However, our previous study has revealed that a small proportion of Escherichia coli could continually carry chromosomally-encoded mcr-1. The chromosomally-encoded events, indicated the existence of stabilized heritage of $\mathrm{mcr}^{-1}$ and revealed a potential threat in the antimicrobial stewardship interventions, are yet to be investigated. In this study, we systematically investigated the genetic basis of chromosomally-encoded $\mathrm{mcr}^{-1}$ in prevalence and potential mechanisms of lineage, plasmid, insertion sequence, and phage. Our results demonstrated that the emergence of chromosomally-encoded mcr-1 could originate from multiple mechanisms, but mainly derived through the recombination of ISAp/1/Tn6330. We reported a specific transmission mechanism, which is a phage-like region without lysogenic components, could associate with the emergence and stabilization of chromosomally-encoded mcr-1. These results highlighted the potential origin and risks of chromosomally-encoded mcr-1, which could be a heritable repository and thrive again when confronted with new selective pressures. To the best of our knowledge, this is the first study to systematically reveal the genomic basis of chromosomally-encoded $\mathrm{mcr}^{-1}$, and report a specific transmission pattern involved in phage-like region. Overall, we demonstrate the origin mechanisms and risks of chromosomally-encoded mcr-1. It highlights the need of public attention on chromosome-encoded $\mathrm{mcr}-1$ to prevent from its reemergence.
\end{abstract}

Keywords: mcr-1, Colistin, Antimicrobial resistance, Genomic pattern, Chromosome, Insertion sequence, Phage

\section{Short report}

The emergence and rapid dissemination of plasmidmediated mobile colistin resistance gene $(m c r-1)$ have become a severe threat to public health [1]. The predominant carriers of $m c r-1$ were IncX4, IncI2, and IncHI2 plasmids, which are transferable and adaptive

*Correspondence: tiangb@mail.sysu.edu.cn

1 Department of Microbiology, Zhongshan School of Medicine, Sun Yatsen University, 74 Zhongshan 2nd Road, Guangzhou 510080, China

Full list of author information is available at the end of the article plasmid types with broad host range and contributed to the spread of $m c r-1$ among various sources and bacterial species [2-4]. Besides, recombination of transposons, especially Tn6330 (ISApl1-mcr-1-pap2-ISApl1), the primary vehicle for transmission of $m c r-1$, and phage-like sequences enable $m c r-1$ to transfer across plasmids and isolates. Such contributed factors facilitated high $m c r-1$ prevalence in several sources around the world, pushing local governments in Europe, Brazil and China to prohibit the use of colistin as growth promoter additive for livestock [5-8]. 
Accumulated evidence showed that banning of colistin in animal feed efficiently restricted $m c r-1$ prevalence, not only in animals but also in humans and the whole ecosystem in China [2-4]. However, our previous study showed that a low proportion of Escherichia coli carrying chromosomally-encoded mcr-1 continually existed in the ecosystem [4], which was sporadically reported by other studies as well [9-11]. On account of the plasmid that could be lost under certain circumstances due to instability, the chromosomally-encoded events could stabilize the heritage of $m c r-1$, threatening the intervention of colistin stewardship. In current study, we systematically investigate the epidemiological and genomic characterizations of $E$. coli population with chromosomally-encoded $m c r-1$.

Based on our previous large-scale epidemiological study from 2016 to 2018 in Guangzhou, China [4], we identified 24 (3.5\%) out of $688 \mathrm{mcr}$-1-positive $E$. coli isolates with the chromosomally-encoded $m c r-1$ (Table 1 ). The prevalence of chromosomally-encoded $\mathrm{mcr}$ - 1 -positive E. coli was from 0 to $9.8 \%$ for each source and from 2.2 to $4.8 \%$ for each epoch, indicating that the chromosomally-encoded $m c r-1$ was at a low prevalence state in different dimensions (Table 1). Additionally, the comparison of prevalence for chromosomally-encoded $\mathrm{mcr}-1$ between different niches or epochs showed no significant difference (Fisher's exact test, $\mathrm{p}>0.05$ for each comparison), suggesting that the emergence of chromosomally-encoded $m c r-1$ was sporadic without temporal or source-specific signals.

To systematically illustrate the genomic basis of chromosomally-encoded $m c r-1$-positive $E$. coli population, we collected other $30 \mathrm{E}$. coli genomes with chromosomally-encoded $m c r-1$ from published literature for subsequent analysis (Additional file 1: Table S1). Through in silico multilocus sequence typing (MLST) assignment, 32 different sequence types (STs) within 10 ST complexes were determined (Fig. 1). The most common ST among chromosomally-encoded mcr-1-positive $E$. coli isolates was ST10 $(\mathrm{n}=10,18.5 \%)$, which is consistent with the main host for plasmid-mediated $m c r-1$ on $E$. coli species $[3,4,12]$. The phylogeny demonstrated two sequence clusters (SCs), except for two isolates which were distinct from two SCs as the outgroup (Fig. 1). The sources and serotypes of these genomes were scattered on the phylogeny, suggesting that the emergence of chromosomally-encoded $m c r-1$ was random without sourceor lineage-based specificity (Fig. 1). Since most of the chromosomally-encoded $m c r-1$-positive $E$. coli isolates have been identified in China $(\mathrm{n}=40,74.1 \%)$, which was attributed to the extensive screening of $m c r-1$ in China, the associations between locations and SCs was ambiguous (SC1 [11/16] vs SC2 [29/36], Fisher's exact test, $\mathrm{p}=0.49)$.

The $m c r-1$ gene was initially found on plasmids in Enterobacteriaceae and on a transposon Tn6330, prompting that the chromosomally-encoded $m c r-1$ could come from recombination of plasmid segments or transposition of Tn6330 [13-15]. Therefore, we investigated the plasmidome of 54 genomes to illustrate the potential origin of chromosomally-encoded $m c r-1$. We identified 33 plasmid Inc types among all isolates, and the results showed that the most common Inc type was $\operatorname{IncFIB}(\mathrm{K})$ $(45.8 \%, \mathrm{n}=22)$, followed by IncColRNAI $(43.8 \%, \mathrm{n}=21)$, IncHI1 $(33.3 \%, \mathrm{n}=16)$, IncX1 $(31.3 \%, \mathrm{n}=15)$, IncFIB (AP001918) $(27.1 \%, n=13)$, and IncY $(20.8 \%, n=10)$. Remarkably, the common Inc types of $m c r-1$-harboring plasmids, such as IncX4, IncI2, IncHI2, and IncpO111 $[1,3,4,12]$, were rarely detected among these isolates (Fig. 1), indicating that the chromosomally-encoded $\mathrm{mcr}$ 1 may derive from ISApl1/Tn6330 through transposition, but not from the plasmid.

We subsequently analyzed the genetic context of $\mathrm{mcr}$ 1 for each isolate to investigate the genetic model of chromosomally-encoded $m c r-1$, except seven isolates were excluded due to short $m c r-1$-harboring contigs.

Table 1 Prevalence of chromosomally-encoded $\mathrm{mcr}$-1 among $688 \mathrm{mcr}$-1-positive $E$. coli isolates

\begin{tabular}{lcccc}
\hline Sample source & \multicolumn{2}{l}{ Epoch (Oct 1 to Dec 31) } & \multicolumn{1}{c}{ Total } \\
\cline { 2 - 4 } & $\mathbf{2 0 1 6}$ & $\mathbf{2 0 1 7}$ & $\mathbf{2 0 1 8}$ & $2.5 \%(5 / 199)$ \\
\hline Pig & $3.8 \%(3 / 78)$ & $0 \%(0 / 63)$ & $3.4 \%(2 / 58)$ & $0 \%(0 / 8)$ \\
Healthy human carrier & $9.8 \%(6 / 61)$ & $4.0 \%(3 / 75)$ & $0 \%(0 / 9)$ & $2.7 \%(3 / 110)$ \\
Colonized patient & $5.0 \%(3 / 60)$ & $0 \%(0 / 41)$ & $0 \%(0 / 11)$ & $0 \%(0 / 55)$ \\
Infected patient & $0 \%(0 / 27)$ & $3.9 \%(2 / 51)$ & $0 \%(0 / 2)$ & $5.6 \%(6 / 107)$ \\
Food & $7.4 \%(4 / 54)$ & $4.5 \%(1 / 22)$ & $0 \%(0 / 1)$ & $1.4 \%(1 / 73)$ \\
Environment & $0 \%(0 / 50)$ & $2.2 \%(6 / 269)$ & $2.2 \%(2 / 89)$ & $3.5 \%(24 / 688)$ \\
Total & $4.8 \%(16 / 330)$ & & &
\end{tabular}

Data are $\%(n / N)$ 


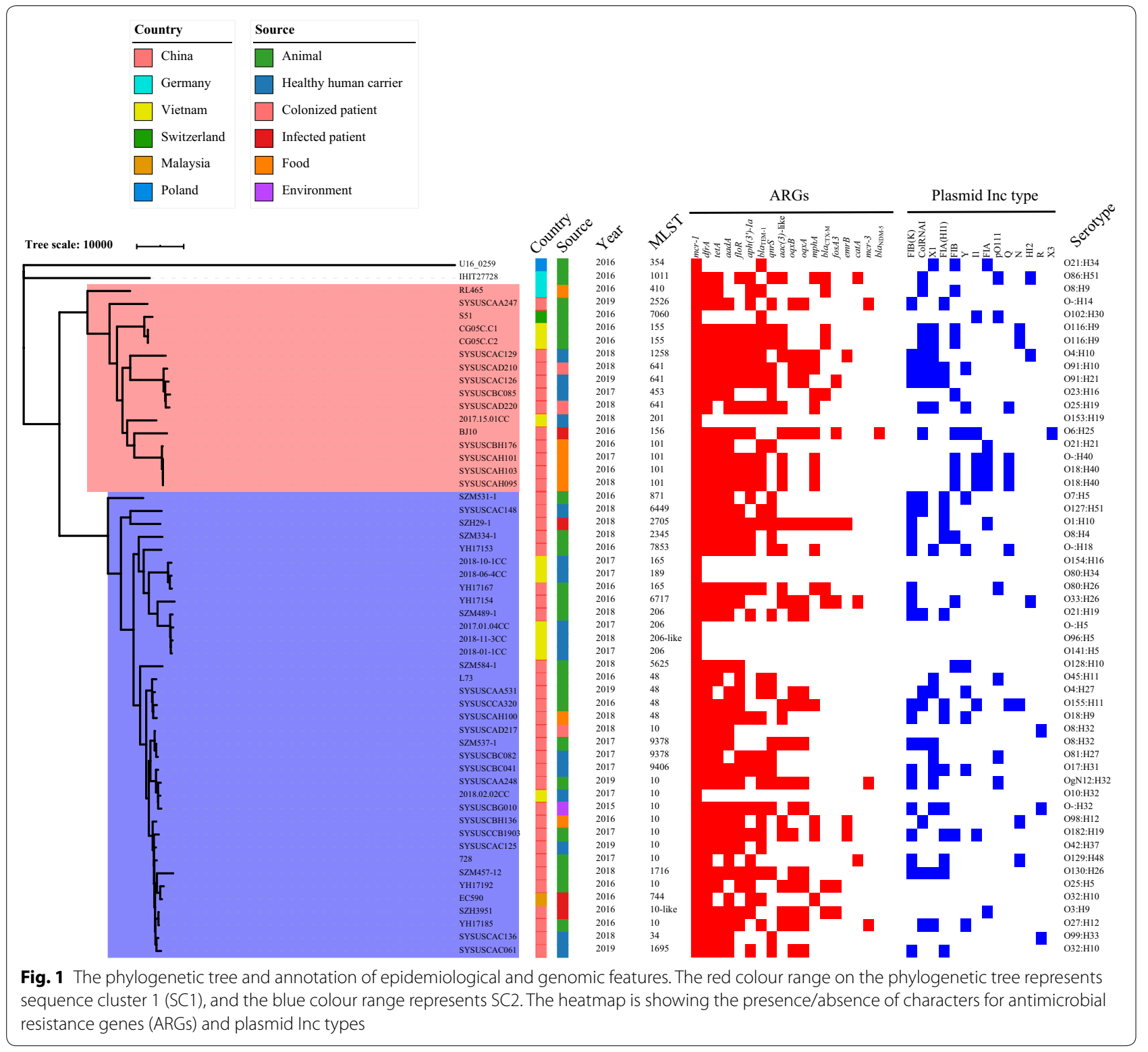

We found that most of the $m c r-1$ genes $(93.6 \%, 44 / 47)$ were flanked by ISApl1, comprising 24 isolates harboring upstream ISApl1 and 20 isolates carrying composite Tn6330, which complied with the hypothesis of transposition-mediated chromosome insertion.
By mapping the insertion site onto the chromosome of $E$. coli MG1655, we noted that the distribution of chromosomally-encoded $m c r-1$ insertion sites was sporadic (Fig. 2a). Thirty-seven clusters of $m c r$-1-harboring segments were generated based on sequence clustering analysis (Fig. 2a), which included three

(See figure on next page.)

Fig. 2 The insertion site and genomic patterns of chromosomally-encoded mcr-1. a The insertion patterns mapped to the Escherichia coli str. K-12 substr. MG1655 (Accession: NC_000913.2). The ring colored with orange represents the genome sequence of Escherichia coli str. K-12 substr. MG1655. The number in the outmost represents the order for each pattern, which showed in $\mathbf{b}, \mathbf{c}$ and Additional file 2: Figure S1. b The genetic structure of chromosomally-encoded $\mathrm{mcr}-1$ patterns which included more than one isolate. $\mathbf{c}$ The genetic structure of chromosomally-encoded mcr-1 which located on an integrative element region and a plasmid-like region 
$\mathbf{a}$

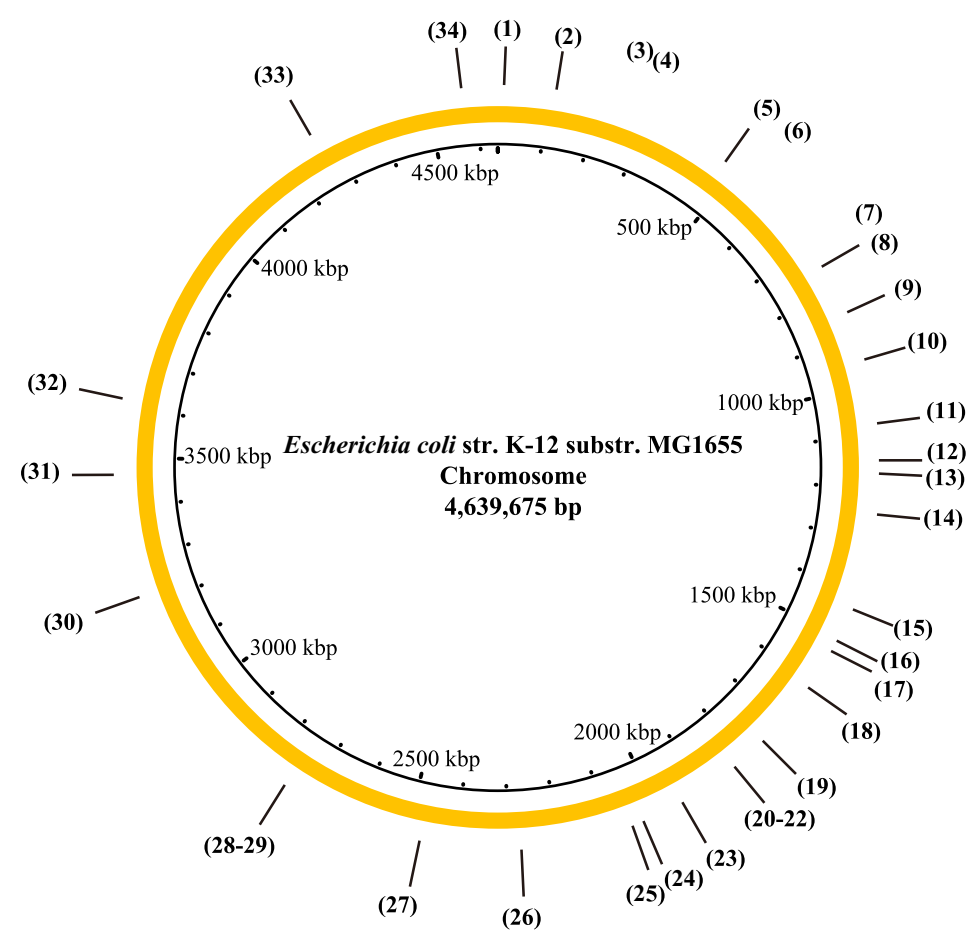

b

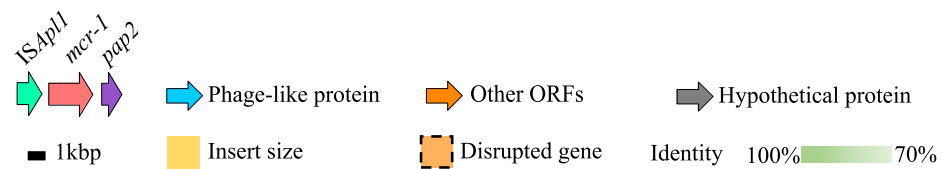

(16)

E. coli str.K-12 substr.MG1655

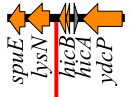

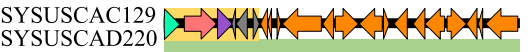

SYSUSCBC 0412 a SYSUSCAH095 SYSUSCAH1012

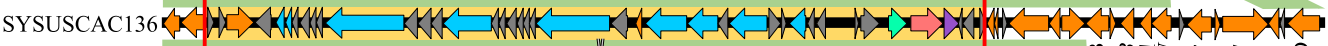
SYSUSCAA531 2 YYSUSCAH100

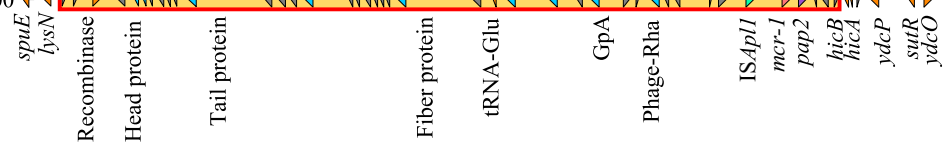
(4) $\quad$ CG05C.C1
andith
焉
(31) $\mathrm{SYSUSCBC082}$ L73

c

(36) SYSUSCCA320

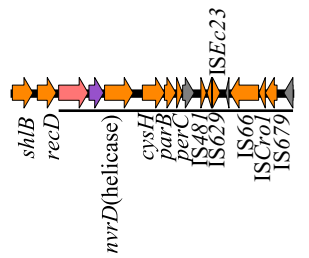

(37)

SYSUSCAA248

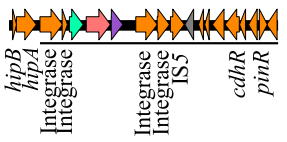


clusters involving more than one isolates (Fig. 2b) and 34 clusters only containing a single isolate (Additional file 2: Figure S1). The most common genetic pattern of chromosomally-encoded mcr-1 (19.1\%, 9/47) involves in an insertion segment in size of $\sim 25.7 \mathrm{~kb}$, containing an incomplete phage-like region (score $=40$ for phage Vibrio 12B8 [NC_021073] by PHASTER) and a truncated Tn6330 (ISApl1-mcr-1-pap2), which was inserted into the E. coli genome between lysN and hicB (toxin-antitoxin system) loci (Fig. 2b). The incomplete phage-like region only contains head, tail, and fiber protein, and lacks some necessary functional components (Fig. 2b), which seems unfunctional under current conditions. We used BLASTn to search this phage-like sequence in NCBI non-redundant nucleotide database, and the results showed that only five sequences, which are located on E. coli chromosome, were identified with $\geq 60 \%$ coverage and $\geq 90 \%$ identity, indicating the correlation between chromosomally-encoded $m c r-1$ and such phage-like region. Collectively, we heuristically concluded that such a phage-like region could mediate the emergence of chromosomally-encoded $m c r-1$, and then the phage may lose the lysogenic components, stabilization the genetic inheritance of chromosomally-encoded $\mathrm{mcr}$ 1. Additionally, the $m c r-1$ of two isolates showed the insertion of $m c r-1$ located on an integrative element region and a plasmid segment respectively, suggesting that chromosomally-encoded $m c r-1$ could be derived from the integration of the integrative region and plasmid segment (Fig. 2c).

In conclusion, our study comprehensively investigated the genetic basis of chromosomally-encoded mcr-1 in prevalence and potential mechanisms of lineage, plasmid, insertion sequence, and phage. Our results showed that chromosomally-encoded $\mathrm{mcr}-1$ was mainly derived from ISApl1 insertion in genomic locations sporadically. Notably, we reported a new transmission mechanism, a phage-like region without functional components, could associate with the emergence and stabilization of chromosomally-encoded $m c r-1$. The chromosomally-encoded $m c r-1$ in current situations seems not a severe threat for public health, however, it could be a heritable repository and thrive again if the new selective pressure emerges, because the chromosome-mediated antimicrobial resistance genes (ARGs) might be conferred with genetic sustainability. In-depth investigations are needed to illustrate the genomic and epidemiological dynamics of chromosomally-encoded $m c r-1$, which may be changed after the approval of colistin in human clinical therapeutics in China [16].

\section{Literature searching}

We searched PubMed using the terms of " $m c r-1$ " [MeSH] /[All Fields] AND "chromosome" [MeSH]/[All Fields] AND "Escherichia coli" [MeSH]/[All Fields] for articles published before $1^{\text {th }}$ October 2020, and identified 20 publications, including 30 available E. coli genomes with chromosome-mediated $\mathrm{mcr}-1$ (Additional file 3: Figure S2).

\section{Bioinformatic analysis}

Antimicrobial resistance genes screening, plasmid incompatibility typing and serotype identification were performed by Center for Genomic Epidemiology (http://www.genomicepidemiology.org/). Multilocus sequence typing (MLST) was assigned using Enterobase (http://enterobase.warwick.ac.uk/). Prophage prediction was implemented by PHASTER [17]. The phylogeny was constructed using RAxML v8.2 with GTR+G model and 1000 bootstrap [18] based on core genome single-nucleotide polymorphisms (cgSNPs) produced by Roary v3.11.2 and snp-site v2.4.1 [19]. Population structure was assessed using cgSNPs with hierBAPS [20]. The chromosome map was drawn by BRIG v0.95 and marked with insertion pattern manually by Easyfig v2.2.2 [21, 22]. The sequence clustering was performed by CD-HIT-EST [23].

\section{Statistical analysis}

The significance of prevalence variation of chromosomally-encoded $m c r-1$ between niches and epochs were tested by Fisher's exact test using Statistical Package for the Social Sciences (SPSS), version 20.0.

\section{Supplementary information}

Supplementary information accompanies this paper at https://doi. org/10.1186/s13099-020-00393-2.

\section{Additional file 1: Table S1.}

Additional file 2: Figure S1. The genetic structure of chromosomallyencoded $\mathrm{mcr}-1$ patterns which included only one isolate. The number for each pattern was identical to Fig. 2a.

Additional file 3: Figure S2. Flow diagram of the study selection process. Additional file 4: Appendix.

\section{Abbreviations}

mcr-1: Mobile colistin resistance gene; MLST: Multilocus sequence typing; STs: Sequence types; SCs: Sequence clusters; cgSNPs: Core genome single-nucleotide polymorphisms; ARG: Antimicrobial resistance gene; Inc: Incompatibility.

\section{Acknowledgements}

We acknowledge Ms. Lujie Liang (Department of Microbiology, Zhongshan School of Medicine, Sun Yat-sen University) for assistance in writing of the manuscript. 


\section{Authors' contributions}

CS, GT and YD designed the study. CS and FM did the literature searching. CS, LZ, FM and GZ collected the data and genomes from NCBI database. CS and FM analyzed the genome data and visualized the results. CS write the draft manuscript. GT and MAE-GE-SA reviewed and edited the final manuscript. All author (except YD) contributed to sample collection and data collection in epidemiological study. All authors reviewed, revised and approved the final submission. All authors read and approved the final manuscript.

\section{Funding}

This work was supported by the National Natural Science Foundation of China (Grant Numbers 81830103, 81722030), China Postdoctoral Science Foundation (BX20200394), National Key Research and Development Program (Grant Number 2017ZX10302301), Guangdong Natural Science Foundation (Grant Number 2017A030306012), Project of high-level health teams of Zhuhai at 2018 (The Innovation Team for Antimicrobial Resistance and Clinical Infection), 111 Project (Grant Number B12003), Open project of Key Laboratory of Tropica Disease Control (Sun Yat-sen University), Ministry of Education (Grant Number 2020kfkt04, 2020kfkt07), Innovative Development Program for Outstanding Graduate Students (No. 19ykyj557), and The Science and Technology Planning Project of Guangdong (2017A020215017).

\section{Availability of data and materials}

The datasets generated and analysed during the current study are available in the NCBI GenBank repository. The accession number for each genome can be obtained in Additional file 4: Appendix material.

\section{Ethics approval and consent to participate}

Not applicable.

\section{Consent for publication}

Not applicable.

\section{Competing interests}

The authors declare that they have no competing interests.

\section{Author details}

1 Department of Microbiology, Zhongshan School of Medicine, Sun Yat-sen University, 74 Zhongshan 2nd Road, Guangzhou 510080, China. ${ }^{2}$ Key Laboratory of Tropical Diseases Control (Sun Yat-sen University), Ministry of Education, Guangzhou 510080, China. ${ }^{3}$ Department of Clinical Laboratory Medicine, Third Affiliated Hospital of Guangzhou Medical University, Guangzhou, China. ${ }^{4}$ Department of Microbiology and Immunology, Faculty of Pharmaceutical Sciences and Drug Manufacturing, Misr University for Science and Technology (MUST), Cairo, Egypt. ${ }^{5}$ University of Pittsburgh School of Medicine, Pittsburgh, PA, USA. ${ }^{6}$ Departments of Microbiology and Infectious Diseases, Fujita Health University School of Medicine, Toyoake, Aichi, Japan. ${ }^{7}$ Department of Clinical Laboratory, Sun Yat-sen Memorial Hospital, Sun Yat-sen University, Guangzhou, China. ${ }^{8}$ Department of Clinical Laboratory, Guangdong Provincial People's Hospital/Guangdong Academy of Medical Sciences, Guangzhou 510080, Guangdong, China. ${ }^{9}$ Department of Clinical Laboratory, The First Affiliated Hospital of Sun Yat-Sen University, Guangzhou 510080, China. ${ }^{10}$ School of Laboratory Medicine, Chengdu Medical College, Chengdu 610500, China.

${ }^{11}$ Department of Neonatal Surgery, Guangzhou Women and Children's Medical Center, Guangzhou, China. ${ }^{12}$ School of Medicine, Xizang Minzu University, Xianyang 712082, Shaanxi, China.

Received: 18 September 2020 Accepted: 17 November 2020

Published online: 28 November 2020

\section{References}

1. Liu YY, Wang Y, Walsh TR, Yi LX, Zhang R, Spencer J, Doi Y, Tian GB, Dong BL, Huang XH, Yu LF, Gu DX, Ren HW, Chen XJ, Lv LC, He DD, Zhou HW, Liang ZS, Liu JH, Shen JZ. Emergence of plasmid-mediated colistin resistance mechanism MCR-1 in animals and human beings in China: a microbiological and molecular biological study. Lancet Infect Dis. 2016;16:161-8.

2. Wang Y, Xu C, Zhang R, Chen Y, Shen Y, Hu F, Liu D, Lu J, Guo Y, Xia X, Jiang J, Wang X, Fu Y, Yang L, Wang J, Li J, Cai C, Yin D, Che J, Fan R,
Wang Y, Qing Y, Li Y, Liao K, Chen H, Zou M, Liang L, Tang J, Shen Z, Wang S, Yang X, Wu C, Xu S, Walsh TR, Shen J. Changes in colistin resistance and $\mathrm{mcr}-1$ abundance in Escherichia coli of animal and human origins following the ban of colistin-positive additives in China: an epidemiological comparative study. Lancet Infect Dis. 2020. https://doi. org/10.1016/S1473-3099(20)30149-3

3. Jiang $Y$, Zhang $Y$, Lu J, Wang $Q$, Cui Y, Wang Y, Quan J, Zhao D, Du X, Liu H, Li X, Wu X, Hua X, Feng Y, Yu Y. Clinical relevance and plasmid dynamics of mcr-1-positive Escherichia coli in China: a multicentre case-control and molecular epidemiological study. Lancet Microbe. 2020;1:e24-33.

4. Shen C, Zhong L-L, Yang Y, Doi Y, Paterson DL, Stoesser N, Ma F, ElSayed Ahmed MAE-G, Feng S, Huang S, Li H-Y, Huang X, Wen X, Zhao Z, Lin M, Chen G, Liang W, Liang Y, Xia Y, Dai M, Chen D-Q, Zhang L, Liao K, Tian GB. Dynamics of $\mathrm{mcr}$-1 prevalence and $\mathrm{mcr}$-1-positive Escherichia coli after the cessation of colistin use as a feed additive for animals in China: a prospective cross-sectional and whole genome sequencing-based molecular epidemiological study. Lancet Microbe. 2020;1:e34-43.

5. Walsh TR, Wu Y. China bans colistin as a feed additive for animals. Lancet Infect Dis. 2016;16:1102-3.

6. Miguela-Villoldo P, Hernandez M, Moreno MA, Rodriguez-Lazaro D, Quesada A, Dominguez L, Ugarte-Ruiz M. National colistin sales versus colistin resistance in Spanish pig production. Res Vet Sci. 2019;123:141-3.

7. Monte DF, Mem A, Fernandes MR, Cerdeira L, Esposito F, Galvao JA, Franco B, Lincopan N, Landgraf M. Chicken meat as a reservoir of colistinresistant Escherichia coli strains carrying $\mathrm{mcr}-1$ genes in South America. Antimicrob Agents Chemother. 2017;61:e02718.

8. Duggett NA, Randall LP, Horton RA, Lemma F, Kirchner M, Nunez-Garcia J, Brena C, Williamson SM, Teale C, Anjum MF. Molecular epidemiology of isolates with multiple mcr plasmids from a pig farm in Great Britain: the effects of colistin withdrawal in the short and long term. J Antimicrob Chemother. 2018;73:3025-33.

9. Li R, Yu H, Xie M, Chen K, Dong N, Lin D, Chan EW, Chen S. Genetic basis of chromosomally-encoded $\mathrm{mcr}-1$ gene. Int J Antimicrob Agents. 2018;51:578-85.

10. Lu XY, Xiao X, Liu Y, Li Y, Li RC, Wang ZQ. Chromosome-mediated mcr-1 in Escherichia coli strain L73 from a goose. Int J Antimicrob Agents. 2019;54:99-101.

11. Peng Z, Hu ZZ, Li ZG, Li XS, Jia CY, Zhang XX, Wu B, Chen HC, Wang XR. Characteristics of a colistin-resistant Escherichia coli ST695 harboring the chromosomally-encoded mcr-1 gene. Microorganisms. 2019;7:558.

12. Wang S, Shen J. Active surveillance of the spread of mcr-1-positive Ecoli. The Lancet Microbe. 2020;1:e4-5.

13. Snesrud E, He S, Chandler M, Dekker JP, Hickman AB, McGann P, Dyda F. A model for transposition of the colistin resistance gene $\mathrm{mcr}-1$ by ISAp/1. Antimicrob Agents Chemother. 2016;60:6973-6.

14. Li R, Chen K, Chan EW, Chen S. Characterization of the stability and dynamics of Tn6330 in an Escherichia coli strain by nanopore long reads. J Antimicrob Chemother. 2019;74:1807-11.

15. Snesrud E, McGann P, Chandler M. The birth and demise of the ISAp/1mcr-1-ISAp/1 composite transposon: the vehicle for transferable colistin resistance. Mbio. 2018;9:e02381.

16. Huang H, Dong N, Shu L, Lu J, Sun Q, Chan EW, Chen S, Zhang R. Colistinresistance gene $\mathrm{mcr}$ in clinical carbapenem-resistant Enterobacteriaceae strains in China, 2014-2019. Emerg Microbes Infect. 2020;9:237-45.

17. Arndt D, Marcu A, Liang Y, Wishart DS. PHAST, PHASTER and PHASTEST: tools for finding prophage in bacterial genomes. Brief Bioinform. 2019;20:1560-7.

18. Stamatakis A. RAxML version 8: a tool for phylogenetic analysis and postanalysis of large phylogenies. Bioinformatics. 2014;30:1312-3.

19. Page AJ, Cummins CA, Hunt M, Wong VK, Reuter S, Holden MTG, Fookes M, Falush D, Keane JA, Parkhill J. Roary: rapid large-scale prokaryote pan genome analysis. Bioinformatics. 2015:31:3691-3.

20. Tonkin-Hill G, Lees JA, Bentley SD, Frost SDW, Corander J. RhierBAPS: an $\mathrm{R}$ implementation of the population clustering algorithm hierBAPS. Wellcome Open Res. 2018;3:93.

21. Sullivan MJ, Petty NK, Beatson SA. Easyfig: a genome comparison visualizer. Bioinformatics. 2011;27:1009-10. 
22. Alikhan NF, Petty NK, Ben Zakour NL, Beatson SA. BLAST Ring Image Generator (BRIG): simple prokaryote genome comparisons. BMC Genomics. 2011;12:402.

23. Fu L, Niu B, Zhu Z, Wu S, Li W. CD-HIT: accelerated for clustering the nextgeneration sequencing data. Bioinformatics. 2012;28:3150-2.

\section{Publisher's Note}

Springer Nature remains neutral with regard to jurisdictional claims in published maps and institutional affiliations.
Ready to submit your research? Choose BMC and benefit from:

- fast, convenient online submission

- thorough peer review by experienced researchers in your field

- rapid publication on acceptance

- support for research data, including large and complex data types

- gold Open Access which fosters wider collaboration and increased citations

- maximum visibility for your research: over $100 \mathrm{M}$ website views per year

At BMC, research is always in progress.

Learn more biomedcentral.com/submissions 